\title{
A brief discussion of mechanism of butylphthalide in treatment of ischemic cerebrovascular disease and its potential clinical applicationin treatment of multiple sclerosis
}

\begin{abstract}
A Chinese traditional medicine Butylphthalide (NBP) has been proved effects in ischemic cerebrovascular disease. Here we discuss the mechanism of NBP in treatment of ischemic stroke, and find its capability in penetrating and modulating Blood-brain barrier (BBB), reducing harmful inflammation and formation of scars in injury CNS tissue, which implicate the potential application of NBP in treatment of Multiple Sclerosis (MS).
\end{abstract}

Keywords: butylphthalide, blood-brain barrier, neovascularization, center nervous system, inflammation, multiple sclerosis, treatment, ischemic cerebrovascular disease
Volume 5 Issue 6 - 2016

\author{
Xin Wang,' Yue Zhang, ${ }^{2}$ Guojun Tan ${ }^{2}$ \\ 'ACURE Biotechnology, Inc. Ellicott City, USA \\ 2Department of Neurology, The Second Hospital of Hebei \\ Medical University. Shijiazhuang, China
}

Correspondence: Xin Wang, ACURE Biotechnology, INC., Ellicott City, Maryland, USA, Email xwang354@gmail.com

Received: April 26, 2016 | Published: May 18, 2016

\section{Introduction}

Butylphthalide (NBP) is a group of new drugs isolated from celery's seeds, originally used for anti-hypertensionin traditional Chinese medicine, ${ }^{1}$ has been developed for anti-ischemia treatment in brain infarction. ${ }^{2}$ Its function in improvement of outcome of cerebral ischemia infarction had been approved. ${ }^{3,4}$ More and more patients get benefits from administrating Butylphthalide. However, the working mechanism of Butylphthalides is far unknown. Here, we literately review the research achievement so far that are related to application of Butylphthalides in cerebrovascular disease in vivo and in vitro. Surprisingly, we found that the metabolites of butylphthalidemay pass through blood-brain barrier, ${ }^{5}$ and butylphthalide may protect the integrity of $\mathrm{BBB}$ and reduce the permeability of BBB injured by reperfusion following hypoxic condition of brain tissue, thereby, reduce the inflammation and water content of brain, prevent brain edema. ${ }^{6}$ Understanding the working mechanism of Butylphthalide will widen the future application of Butylphthalides and increase clinic efficacy on treatment and diagnosis of vascular and inflammation diseases in center nervous system (CNS).

Mechanism of Butylphthalides in treatment of cerebral ischemia infarction

I. Butylphthalide modulate inflammation by increasing circulation endothelium progenitor cells (EPCs) ${ }^{7}$ and reducing the infiltration of neutrophils to injured post-ischemic brain tissue. Yip HK et al. ${ }^{8}$ studied the prognostic value of circulating EPCs in acute ischemia stroke (IS) with 138 consecutive patients and found that circulating EPCs is the independent factor for prognosis of outcome of acute IS. The bone marrowderived EPCs migrate following the blood stream to injured lesion, differentiate to mature endothelial cells for angiogenesis in situ. It has been reported that Butylphthalide administration increase the level of EPCs in blood stream that associate with the outcome of IS in a clinical study with 170 stroke patients. The results suggest that somehow Butylphthalide increase EPCs passing through BBB to ischemic lesions for injured brain tissue healing process. On the other hand, Butylphthalide inhibit the infiltration of neutrophils to injured lesions of ischemic-reperfusion after transient focal cerebral ischemia in rats that decrease the inflammation. ${ }^{9}$ Therefore, Butylphthalide improve the healing of post-ischemic brain tissue through migrating immune healing cells.

II. Butylphthalidepromote angiogenesis with increasing expression of pro-angiographic factors:

A chick embryonic chorioallantoic membrane assay shows the profound effect of angiogenesis of DL-NBP with increased expression of pro-angiographic factors: growth factors, vascular endothelial growth factor (VEGF), VEGF receptor and basic fibroblast growth factor (bFGF). ${ }^{10}$ In a middle cerebral artery occlusion (MCAO) model with Stroke-prone renovascular hypertensive rats, NBP treatment rescues brain tissue after ischemic stroke by enhancing angiogenesis with up-regulation of VEGF and hypoxia inducible factor 1 (HIF1) alpha. ${ }^{11}$ Therefore, angiogenesis induced by pro-angiographic immune factors play an important role in the mechanism of NBP in treatment of ischemic cerebrovascular diseases.

III. Butylphthalideinhibit the activation of micogliaand astrocytes, reduce pro-inflammation factors produced by microglia and astrocytes in injured brain territoryto decrease the scar area, improve the restoration of ischemic lesions of brain tissue. The astrocytes and microglia-mediated neuroinflammation play a central role in pathogenesis of ischemic brain injury by releasing inflammatory mediators. Over-activated microglia releasing excessive pro-inflammatory factors cause over immune reaction in the lesions, which lead to less function restoration from ischemic stroke. Suppression of activation of microglia is a therapeutic strategy in improving the outcome of stroke. ${ }^{12}$ Astrocytes also modulate the permeability of BBB by interacting with endothelial cells with released immune mediators. ${ }^{13}$ Zhao et al. ${ }^{14}$ found that NBP attenuate neuroinflammation by inhibiting the microglia activation in lipopolysaccharide-treated mice. ${ }^{14}$ In the culture system of primary astrocytes isolated from cerebral cortex of rats, NBP attenuate the inflammatory responses induced by amyloid-beta through inhibiting activation of astrocytes and proinflammatory products. ${ }^{15}$ These results implicate that NBP may improve 
the restoration of neurological function through reducing neuroinflammation mediated by microglia and astrocytes and their pro-inflammation products.

\section{Potential application of butylphthalide in treatment of multiple sclerosis (MS)}

Multiple Sclerosis (MS) is a demyelination disease affecting 2.5 million people worldwide and no cure has been reported up to date. Patients with MS relapse presenta variety of physical, mental neurological disorders, even death as the disease advances. MS is well known as most common autoimmune disease occurring in CNS. The leakage of BBB is a common pathological property in multiple sclerosis (MS).${ }^{16}$ Genetics or environmental factors like infection of microbes combined with immune system disorder induce the dysfunction of BBB and the entry of immune cells and antibodies passing through $\mathrm{BBB}$ into tissue of brain and spine cord. Immune cells invasion and their secreted cytokines, chemokines and antibodies attack the neuron supporting and myelinating cells, such as oligodentrocytes, astrocytes, pericytes and neuroglia, disrupt the myelin sheath of nerve cells, eventually damage the neurons. Between the two relapses, remyelinationmay occur, but unsuccessfully remyelinated lesions form scar-like plaques where permanently lose neurological functions. The overlaps of mechanism of Butylphthalides in treatment of ischemia cerebrovascular diseases with the pathological process of MS lead us to consider the clinical feasibility and efficacy of application of Butylphthalides in treatment of MS. We hypothesis that NBP may reduce pro-inflammation cells, increase mature endothelia cells in the MS lesions to mild the inflammation responses in relapsing phase; NBP may increase angiogenesis with angiographic factors for restoration of neovascularization in MS lesions; NBP may inhibit the pro-immune activation of microglia and astrocytes so that reducing formation of scars, improving remyelinating of neurons. Taken together, the mechanism of NBP implicates great potential in the healing of MS.

Brain and spine cord are known as immune privilege organ in certain ways because blood-brain barrier (BBB), the neuroimmune structure between blood vessels and brain tissue, blocks the 'antigens' and immune cells entering CNS tissue for prevention from inflammation. ${ }^{17}$ In normal condition, there are very few immune cells existing in brain and spine cord tissue. The main elements of BBB structure include tightly connected endothelium cells, basal membrane and astrocytes, terminal of neurons. Depending on the characteristics of the brain specific structure, only brain essential nutrition like glucose, oxygen, small amino acids and so on can pass through this brain-specific structure to provide the original energy materials for brain tissue survival and brain specific functions. This feature protects CNS tissue but at same time bring difficulties to treatment and precise diagnosis of CNS diseases. For instance, neuron protective drugs hardly locate to brain tissue become functional. Therefore, the studies about BBB have significant meaning to neurological diseases. The discovery of capability of Butylphalide and its metabolites in mild passing through BBB not only lighten the way for improvement of outcome of ischemic cerebrovascular diseases and MS, but also give us hope for find cures for other deadly CNS disorders like degenerative diseases and tumor by combining with nanotechnology. ${ }^{18}$

\section{Acknowledgments}

None.

\section{Conflicts of interest}

The authors declare no conflicts of interest.

\section{Funding}

None.

\section{References}

1. Lu HC. Chinese System of Food Cures:Prevention and Remedies. Sterling Publishing, New York, USA. 1986.

2. Zhang JT. New drugs derived from medicinal plants. Therapie 200;57(2):137-150

3. Du R, Teng JF, Wang Y, et al. Clinical study of Butylphthalide combined with XueShuan Tong on serum inflammatory factors and prognosis effect of patients with cerebral infarction. Randomized controlled trial. Pak J Pharm Sci. 2015;28(5 Suppl):1823-1827.

4. Cui LY, Zhu YC, Gao S, et al. Ninely-day administration of dl-3-nbutylphthalide for acute ischemic stroke:a randomized double-blind trial. Randomized controlled trial. Chin Med J (Engl). 2013;126(18):34053410 .

5. Diao XX, Zhong K, Li XL, et al. Isomer-selective distribution of 3-nbutylphthalide (NBP) hydroxylated metabolites, 3-hydroxy-NBP and 10-hydroxy-NBP, across the rat blood-brain barrier. Acta Pharmacol Sin. 2015;36(12):1520-1527.

6. Chong ZZ, Feng YP. dl-3-n-butylphthalide attenuates reperfusioninduced blood-brain barrier damage after focal cerebral ischemia in rats. Zhongguo Yao Li Xue Bao. 1999;20(8):696-700.

7. Zhao H, Yun W, Zhang Q, et al. Mobilization of circulating endothelia progenitor cells by dl-3-n-Butylphthalide in Acute Ischemic Stroke Patients. J Stroke Cerebrovasc Dis. 2016;25(4):752-760.

8. Yip HK, Chang LT, Chang WN, et al. Level and value of circulating endothelial progenitor cells in patients after acute ischemic stroke. Stroke. 2008;39(1):69-74.

9. $\mathrm{Xu} \mathrm{HL}$, Feng YP. Inhibitory effects of chiral 3-n-butylphthalide on inflammation following focal ischemic brain injury in rats. Acta Pharmacol Sin. 2000;21(5):433-438.

10. Zhang L, Lu L, Chan WM, et al. Effects of DL-3-n-butylphthalide on vascular dementia and angiogenesis. Neurochem Res. 2012;37(5):911919

11. Liao SJ, Lin JW, Pei Z, et al. Enhanced angiogenesis with dl-3-nbutylphthalide treatment after focal cerebral ischemia in RHRSP. Brain Res. 2009;1289:69-78.

12. Yuan Y, Fang $\mathrm{M}, \mathrm{Wu} \mathrm{CY}$, et al. Scutellarin as a potential therapeutic agent for microglia-mediated neuroinflammation in cerebral ischemia. Neuromolecular Med. 2016

13. Abbott NJ. Astrocyte-endothelial interactions and blood-brain barrier permeability. J Anat 2002;200(6):629-638.

14. Zhao CY, Lei H, Zhang Y, et al. L-3-n-Butylphthalide attenuates neuroinflammatory responses by downregulating JNK activation and upregulatingHeme oxygenase-1 in lipopolysaccharide-treated mice. $J$ Asian Nat Prod Res. 2016;18(3):289-302.

15. Wang HM, Zhang T, Huang JK, et al. 3-N-butylphthalide (NBP) attenuates the amyloid-beta-induced inflammatory responses in cultured astrocytes via the nuclear factor-kB signaling pathway. Cell Physiol Biochem. 2013;32(1):235-242.

16. LeVine SM. Albumin and multiple sclerosis. BMC Neurol. 2016;16(1):47.

17. Banks WA. The blood-brain barrier in neuroimmunology:Tales of separation and assimilation. Brain Behav Immun. 2015;44:1-8.

18. Mangraviti A, Gullotti D, Tyler B, et al. Nanobiotechnology-based delivery strategies:New frontiers in brain tumor targeted therapies. $J$ Control Release. 2016. 